\title{
I SERVIZI DI SALUTE MENTALE PER I BAMBINI IN INGHILTERRA (E IN ITALIA) "NON SONO NEANCHE LONTANAMENTE SUFFICIENTI"
}

"I giovani e i bambini soffrono molto di più di quanto la gente pensi. A mio parere, le persone sottovalutano quanto i giovani e $i$ bambini siano influenzati da queste circostanze attuali”.

Ragazzo, 16 anni, risposta a una consulenza online

"Sono stato in lista d'attesa per una consulenza per anni. So che sono tempi difficili per tutti, ma se ci fosse più disponibilità per le consulenze penso che le persone sarebbero più felici"'.

Tim, I5 anni, Merseyside

"Il supporto per la salute mentale nelle scuole deve migliorare molto. Non abbiamo affatto un consulente, solo un'infermiera ogni due settimane. Tutti sono nervosi e penso che le persone trarrebbero molto più beneficio dal parlarne"".

Kim, I6 anni, South Yorkshire

II danno alla salute mentale dei bambini causato dalla pandemia Covid-19 potrebbe durare per anni a meno che i Servizi non vengano migliorati e ampliati, ha avvertito il commissario inglese per la salute dell'infanzia e l'adolescenza'.

Anne Longfield ha affermato, nel suo Rapporto annuale, che la disponibilità di Servizi di salute mentale per i bambini in Inghilterra "non era ancora neanche lontanamente sufficiente a soddisfare i loro bisogni" e che il governo deve riconoscerlo e "andare oltre gli impegni esistenti con nuovi ambiziosi obiettivi per aumentare l'accesso alle cure".

$\mathrm{Ha}$ anche sottolineato che alcuni gruppi di clinici hanno "costantemente sottovalutato la salute mentale dei bambini, ignorato i loro bisogni e non sono riusciti a soddisfare le aspettative del NHS inglese e ne devono ora affrontare le conseguenze".

Si stima che un bambino su sei in Inghilterra abbia un probabile problema di salute mentale e il numero di bambini indirizzati a un aiuto assistenziale è in aumento. Nel 2019-20 questo è salito a 538.564 , con un aumento del $35 \%$ dal 2018 19 e del $60 \%$ dal $2017-18$.

Tuttavia, il numero di bambini che accedono alle cure non aumenta abbastanza rapidamente da soddisfare la domanda. Nel 2019-20 solo 39l.940 bambini hanno avuto una presa in carico (un quarto di quelli che si stima ne abbiano bisogno). Quelli che hanno avuto accesso ai Servizi spesso hanno dovuto aspettare settimane o mesi per il trattamento e solo il $20 \%$ dei bambini ha iniziato entro quattro settimane dalla richiesta.

II rapporto ha anche evidenziato che la spesa per la salute mentale dei bambini è in aumento ma risulta essere inadeguata rispetto ai bisogni e altamente variabile nonostante $i$ numerosi annunci negli anni fatti dal Governo. "In media, le aree del gruppo di committenza clinica locale hanno speso meno dell'।\% del budget complessivo per la salute mentale dei bambini e 14 volte di più per i servizi di salute mentale degli adulti”.

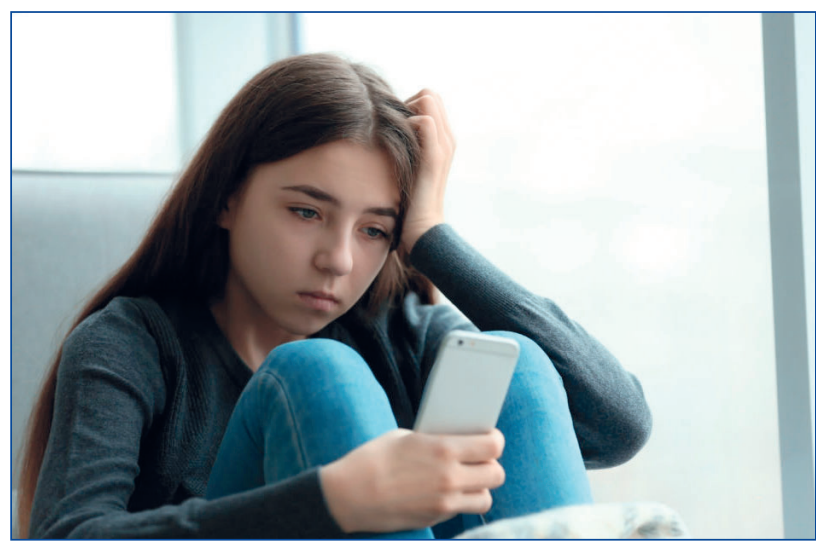

Impatto del Covid- I 9

Sebbene questo rapporto si concentri sul periodo prima che la pandemia prendesse piede (da marzo 2019 a marzo 2020), ci sarebbero prove molto forti che il Covid-19 sta avendo un "impatto significativamente dannoso sulla salute mentale di alcuni bambini e adolescenti”.

Nel luglio 2020 un sondaggio commissionato da NHS Digital ha rilevato che la prevalenza di condizioni di salute mentale clinicamente significative tra i bambini era superiore del $50 \%$ rispetto al precedente rilievo del 2017. Anche se non è certo quanto l'aumento sia stato un risultato diretto del pandemia, i dati precedenti degli ultimi 15 anni hanno dimostrato che l'aumento è normalmente graduale, suggerendo che questo incremento significativo e rapido è una conseguenza del Covid-19. II rapporto afferma: "L'aspirazione nel piano decennale del NHS di fornire supporto a tutti i bambini e adolescenti che necessitano di cure specialistiche entro il 2028 è molto importante. Questa ambizione necessita ora di un chiaro piano di consegna per garantire il raggiungimento di questo obiettivo." Ciò sarà possibile solo se il sistema offerto verrà ampliato per includere il sostegno nelle scuole, online e dal settore del volontariato". È stato chiesto al Governo di impegnarsi a implementare team di supporto per la salute mentale - team integrati che lavorano tra le scuole e il NHS - in ogni regione dell'Inghilterra.

\section{II commento}

Il testo riportato è stato pubblicato sulle pagine del $B M{ }^{2}$, la rivista letta settimanalmente da migliaia di operatori sanitari e persone che sono deputate a decisioni in temi di Salute pubblica.

La situazione inglese è molto simile a quella italiana ma, per quanto ci riguarda, con dati non sempre così precisi o per lo meno discussi in modo partecipe.

Sono di questi giorni, anche in Italia, le grida di allarme di diversi operatori sanitari e di alcune Società scientifiche sullo stato di salute mentale dei bambini e soprattutto adolescenti in tempi di 
pandemia ${ }^{3}$. Si citano in particolare l'importante incremento dei casi di anoressia, di autolesionismo, di tentativi suicidari. Potremmo dire la punta dell'iceberg, quelle situazioni eclatanti e chiaramente "visibili". Ci si accorge che, anche in Italia, i posti letto per il ricovero in ambiente neuropsichiatrico dei bambini e dei ragazzi che ne hanno bisogno di fatto non ci sono. Sono 92 in tutto il Paese. Ci si appoggia dove si può, quasi sempre nei reparti di Pediatria, in una sorta di accoglienza di sostegno (non sempre di progetto cura). Da questo punto di vista sono in sofferenza tutte le Regioni italiane. Sottovalutare l'impatto del Covid-1 9 tra i più giovani (in una situazione già molto critica in termini di personale, Servizi e organizzazione assistenziale per i problemi neuropsichiatrici dell'infanzia e adolescenza) rischia di trasformare un'emergenza sanitaria come quella che stiamo vivendo in una crisi dei diritti dei bambini e dei ragazzi. Inoltre, sebbene sia ancora prematuro tracciare un quadro preciso delle reali conseguenze della pandemia sul benessere mentale dei più piccoli, sappiamo che alcune di queste si manifestano a distanza e i dati disponibili sono già ora preoccupanti ${ }^{4}$.

Stefano Vicari (direttore della NPI dell'Ospedale "Bambino Gesù") in una sua intervista ha dichiarato: "almeno il $20 \%$ adolescenti ha un disturbo mentale, che è stessa percentuale che si ritrova nella della popolazione generale, mentre nell'infanzia siamo al 10\%. Sono $i$ disturbi in assoluto più frequenti, ma la cosa paradossale è che in Pediatria non si studia Psichiatria e quindi la diagnosi è spesso tardiva, oppure $i$ genitori sono lasciati soli e non sapendo dove andare si rivolgono allo psicologo sotto casa"s.

Non possiamo dunque far scorrere il tempo senza definire interventi capaci di mitigare il più possibile tutti gli effetti negativi fin qui riscontrati e quelli, ad oggi, solo ipotizzabili ${ }^{3,4,6}$.

Non è questa la sede per prospettare concretamente quelle che devono essere delle scelte di indirizzo chiaro che mettano la salute (anche mentale) dell'infanzia e adolescenza al centro delle politiche socio-sanitarie del paese e dei singoli territori, cosa da fare coinvolgendo i neuropsichiatri infantili, gli psicologi, i servizi educativi e quelli sociali, il terzo settore, oltre ai pediatri. Certamente deve essere rafforzata la componente preventiva e di individuazione precoce, a partire dall'ambulatorio del pediatra di famiglia e della scuola. Certamente devono essere rafforzati i Servizi territoriali di Neuropsichiatria, oggi gravemente carenti. E certamente potenziata la risposta all'acuzie grave, che sia in regime di ricovero o di accoglienza in Centri specialistici.

Medico e Bambino ha in più occasioni posto al centro del dibattito gli ambiti di priorità sul problema dei bisogni dei bambini e delle famiglie con disturbi neurospichiatrici, anche in ambito formativo ${ }^{7}$. Era inevitabile pensare, come da subito abbiamo cercato di fare, che si sarebbero rese evidenti, al pari di quello che è stato riportato in Inghilterra ${ }^{1,2}$, le conseguenze di alcune inadempienze organizzative e sottovalutazioni di problemi nel contrasto alla pandemia ${ }^{8-10}$.

Sul numero di aprile 2020 di Medico e Bambino si proponeva "P'istituzione di un Gruppo multidisciplinare di coordinamento centrale, nazionale o regionale, che possa orientare gli interventi di salute mentale, predisponendo progetti e programmi coerentemente ai bisogni della popolazione e all'andamento della diffusione dell'epidemia sul territorio nazionale". Non si è visto ancora nulla del genere. Abbiamo sempre detto che non è tempo delle polemiche ma delle "costruzioni" partecipi, solidali e qualificate, prendendo spunto magari da alcune proposte del modello inglese'. Ma è anche tempo di porre al centro del dibattito pratico e propositivo (ma di ora) un'altra concreta "rivoluzione gentile" - che è quella di imparare l'alfabeto con cui le nuove forme del disagio adolescenziale vengono espresse e di forgiare strumenti per agire in un'ottica che coinvolga, oltre agli adolescenti stessi, le famiglie, la scuola e la comunità tutta ${ }^{\prime \prime}$.

Molto dobbiamo fare per (ri)orientare i Servizi sui bisogni di salute mentale dei giovani, Servizi che oggi sono caratterizzati da un elevato livello di frammentazione nei metodi, nei luoghi, nelle modalità di interazione, il che spesso comporta anche la mancata richiesta di aiuto, il che appare paradossale in relazione all'efficacia dei risultati possibili". Le situazioni di "disagio" (temine generico e inappropriato) devono avere diagnosi precise e percorsi terapeutici strutturati (territoriali in primis ma anche ospedalieri per i casi più gravi), al pari di qualsiasi malattia che viene definita come "organica". E solo una rete di professionisti e di servizi lo può fare ${ }^{\prime 2}$. Non di certo il fiorire incontrollato di offerte private e parcellari di assistenza.

Molto dobbiamo fare per porre rimedio (in un bilancio complessivo di scelte prioritarie di salute) ai dati appena pubblicati da Save the Children che ci dicono che in un anno a livello planetario sono stati persi I I 2 miliardi di giorni di istruzione scolastica (soprattutto tra $i$ poveri) e che in Italia esistono disparità importanti tra il Nord e il Sud: i bambini a Milano sono andati in classe I I 2 giorni vs i 48 di quelli di Bari; gli adolescenti di Reggio Calabria appena 35 giorni contro i 75 di Firenze. Sono le due facce di una stessa medaglia.

\section{Bibliografia}

I. Children's Commissioner. The state of children's mental health services 2020-2I. Jan 202I.

2. Mahase E. Covid- 19: Children's mental health services in England are "nowhere near sufficient", says commissioner. BMJ 2021;372: n258. doi: I0.1 I36/bmj.n258.

3. Vicari S, Di Vara S (a cura di). Bambini, adolescenti e Covid-19. L'impatto della pandemia dal punto di vista emotivo, psicologico e scolastico. Erickson editore. Gennaio 202I.

4. Green P. Risks to children and young people during covid-19 pandemic. BMJ 2020;369:m I669. doi: I0.1 I36/bmj.m I669.

5. L'onda lunga del Covid sugli adolescenti? Va affrontata adesso. Intervista a Stefano Vicari (di Sara De Carli). Vita, 19 gennaio 202I. 6. Gruppo di Lavoro su Covid-19 in Pediatria della Regione EmiliaRomagna (RE-CO-PED). Gestione del Covid-19 in età pediatrica: documento di consenso. Medico e Bambino 2021;40(2):85-I0I doi: 10.53 $126 /$ MEB40085.

7. Ventura A, Marchetti F. Appunti di Neuropsichiatra. Medico e Bambino 2020;39(3): I 142-3.

8. Abbracciavento G, Cognini M, Riccio G, Carrozzi M. Covid-19 e salute mentale in età evolut-va. L'urgenza di darsi da fare. Medico e Bambino 2020;39:237-40.

9. Marchetti F. Gli effetti avversi del Covid-19 in età pediatrica: la pandemia secondaria. Medico e Bambino pagine elettroniche 2020;23(6): II5-7.

10. Tamburlini G. L'impatto della pandemia Covid-19 sulla salute globale dei bambini. Medico e Bambino 2020;39(10):629-32.

II. Ruggeri M. Una rivoluzione "gentile" e necessaria per investire sulla salute mentale dei giova-ni. In: prefazione a Monzani E, Meneghelli A, Percudani M (a cura di). Stati mentali a rischio. Riconoscere, prevenire, curare nei giovani. II Pensiero Scientifico Editore, 2019.

12. Centro per la Salute del Bambino, ACP. SEnza confini. Come ridisegnare le cure all'infanzia e all'adolescenza, integrando i servizi, promuovendo l'equità, diffondendo le eccellenze. 2020. https://www.medicoebambino.com/lib/senza_confini.pdf

Federico Marchetti 